\title{
Review of Various Strategies for Gateway Discovery Mechanisms for Integrating Internet-WANET
}

\author{
Shweta Bansal \\ Research scholar \\ Institute of Engineering and \\ Technology, Bhaddal
}

\author{
Pradeep Kumar Gaur \\ Assistant Professor \\ Institute of Engineering and \\ Technology, Bhaddal
}

\author{
Anupma Marwaha, PhD \\ Associate Professor \\ SLIET,Longowal
}

\begin{abstract}
The communication range of devices in a wireless ad hoc network is inherently limited, but it can be enhanced by interconnecting it to a wired network like the internet, thus forming a hybrid wireless network. This interconnection to improve scalability has been achieved by using gateways, which brings the biggest overhead in WANET accessing Internet. So a mobile node in WANET has to find a route to a gateway first to communicate with the Internet host. It requires an efficient gateway discovery mechanism. The discovery and selection of Internet gateway are one of the key challenges to provide inter-connectivity. The gateway discovery mechanisms called proactive, reactive and hybrid are developed to enable mobile nodes to register with internet gateways. But the performance of the network largely depends on the gateway discovery method used and it can be analyzed using various performance metrics such as packet delay, throughput, routing overhead, Bandwidth efficiency etc. This paper focuses on various issues for WANET-Internet integration and their proposed technical solutions by various researchers for different Internet gateway discovery mechanisms to improve the performance.
\end{abstract}

\section{Keywords}

Gateway discovery mechanism, Internet Gateway, routing, WANET

\section{INTRODUCTION}

A wireless ad-hoc network (WANET) is a self-governing and self-directing network consisting of a set of identical mobile nodes which communicate with each other via wireless links. It has no established infrastructure and centralized administration. The network's topology is very much unpredictable and can change quickly. The flexibility, easy deployment, and self-configurability of WANETs are its most important features. The Integration of the Internet with heterogeneous networks viz. WiMAX, ZiGBEE, WLAN etc. increases the sphere of influence of ad hoc networks, as it extends its network coverage and scalability. But as the Internet and WANET architectures are different, therefore various sorts of assumptions are imposed on the structure, topology of the underlying networks and on the communication patterns of their mobile nodes to integrate them into a hybrid network which is very challenging.

An Internet gateway (IG) is required to provide the Internet Connectivity for the nodes in the WANETs to let the user access the global services like Internet. Numerous routing protocols have been projected for WANETs such as AODV, DSR, DSDV, TORA and ZRP etc. but they are for local communication within the WANET. Ad hoc routing protocol needs to be modified before a mobile node in WANET can communicate with the Internet hosts. It requires gateway and thus $\mathrm{MN}$ needs to find a route to a gateway first. But because of scarce network resources, it is the challenge to convey and update mobile nodes about available IGs making least use of the resources. So, an efficient gateway discovery for Ad Hoc networks becomes one of the key elements to improve the performance and make it use in future mobile and wireless networks taking into consideration minimal consumption of the resources. Each mobile node within the WANET must configure globally routable IP address [1] to communicate with the Internet. Once the node enters the WANET, it acquires a temporary address for communication within the WANET. It has to form a globally routable address by discovering and selecting one IG to use its prefix for global communication.

This paper analyzes the solutions proposed by various researchers and their contributions and mechanisms involved for the advancement in technical issues for WANET- Internet integration. In the following sections, we have discussed about the background in section II. Section III discusses some methods of discovering a gateway and routing data towards the gateway. Analysis of Different Internet Connectivity proposals for WANETs is discussed in section IV. Finally, section $\mathrm{V}$ provides conclusion and future scope.

\section{BACKGROUND}

The main objective of IIW is to allow nodes attached to the Internet to share connectivity with WANET nodes, which may be unable to directly access Internet resources.

\subsection{Routing in WANET and Internet}

In WANET, each node of the ad-hoc network acts as a router for all other nodes. Ad Hoc routing protocols [2, 3] are needed to forward data packets from one node to the next until they are received by a destination node.

But Internet has a different infrastructure from that of WANET, with predefined routers and gateways in which nodes communicate with other nodes via these routers. Furthermore, same network ID is shared by all hosts on a particular network, therefore only one entry in the routing table can handle all such hosts. Also, default routes can be used in case no other route exists to a destination.

\subsection{Issues in Internet-WANET Integration} The several important issues that should be considered for Internet-WANET Integration (IIW): 
1. Load Balancing: To avoid gateway nodes to become the bottleneck as they have to face heavy traffic being the one that connect the ad-hoc network to the Internet. Load balancing techniques are needed to share the load.

2. Security: Security is a major concern as any node can join or withdraw from WANET randomly and nodes have no restrictions on their Mobility.

3. Service, address, and location discovery: there exist protocols to provide these services in internet but they need to be extended to service ad hoc networks also connected to the Internet.

4. Addressing: In WANET, a mobile node has to maintain two addresses simultaneously; one to ensure mobility and other to have global connectivity.

5. Routing: Internet uses hierarchical routing while WANET uses flat routing.

6. multi homing and hand offs

\subsection{Solution}

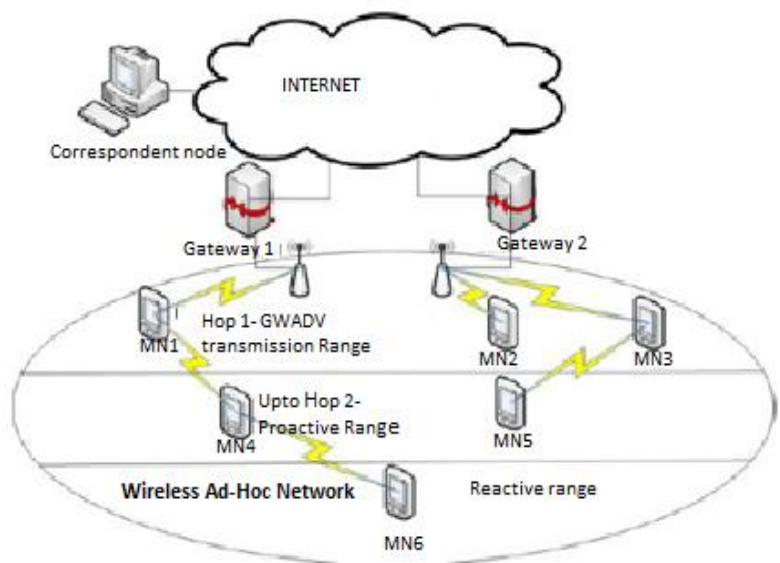

Figure 1. GDM in Heterogeneous Networks for TTL=2

A new node, called an Internet gateway [2], is used to interconnect ad-hoc mobile nodes to the Internet. It may be a static node or a mobile node and is equipped with two wireless interfaces, one as part of the ad-hoc network and the second as a link to the Internet thus acting as a part of both networks simultaneously. Firstly, the Gateway must be discovered by the mobile nodes of the WANET to get connected to the Internet. Three different approaches can be used to achieve this discovery, namely, Proactive, reactive and hybrid.

1) Reactive Gateway Discovery: a mobile node initiates this discovery by broadcasting a message throughout the network for creating or updating route to the Internet.

2) Proactive Gateway Discovery: the gateway itself initiates this discovery by periodically broadcasting its services and IP prefix address throughout the network.

3) Hybrid Gateway Discovery: This approach overcomes the limitations of reactive and proactive approaches. It controls the scope of flooding advertisement and decreases the routing overhead by combining the above two approaches. The nodes using proactive approach are constrained within a certain range and reactive approach is used by nodes outside this range.

\section{SOME METHODS OF DISCOVERING AND ROUTING DATA TOWARDS GATEWAY}

There are various methods of discovering gateway and routing data towards the gateway. Some of these are [4]:

1. If the signal-noise ratio measured by a node on dedicated beacon messages sent by the gateway is above a predetermined threshold, it is considered to have good connectivity with the gateway. Selection of gateway nodes can be centralized or distributed.

2. It includes a step of broadcasting of the advertisement message only to the gateway node(s) including a route to said gateway; and a step of hop-by-hop rebroadcasting of it in the ad hoc network by each of the nodes receiving the message at least to a depth fixed by the gateway (proactive area of the network).

3. It includes a step of extending the proactive area during which at least one node of the ad hoc network rebroadcasts the advertisement message if that node knows of at least one active source in the ad hoc network separated from the gateway by a plurality of hops greater than said depth.

4. As soon as a node commences to send data to the gateway, it becomes an active source, and ceases to be an active source if it does not communicate with the gateway for a predetermined period.

5. Each intermediate node that forwards data sent by an active source to the gateway updates the list of active sources of the network and the number of hops between the active source and the gateway.

6. During extension of the proactive area, for bandwidth efficiency, the advertisement message is sent only to the nodes able to set up a path to an active source.

7. Includes a step of the gateway determining the depth in order to maximize gain taking account of the no. of active sources in the proactive area and of a cost criterion. The gateway holds topological information of the number of connected nodes and their depth.

8. Includes a step in which, the gateway waits for a period (computed by the gateway) between two successive broadcasts of the advertisement message, taking activity and the mobility of the nodes of the network into account. The activity $A_{i}$ in the network can be defined as the ratio between the number $\mathrm{S}$ of active sources and $\mathrm{N}$ number of total nodes in the network. The mobility $\mathrm{M}_{\mathrm{i}}$ is the ratio between the number $M$ of moved nodes in the ad hoc network including nodes that join or quit the network during this period in a period of time and $\mathrm{N}$ number of total nodes.

9. Includes a step of the gateway adjusting the TTL depth intended to distribute the traffic between the gateway and at least one other neighbor gateway.

10. The fixed infrastructure of the hybrid network includes an entity responsible for implementing load balancing adapted to assure good traffic distribution between different gateways. 
11. Includes a step in which the gateway reduces the depth and a period between two successive broadcasts of the advertisement message if the activity of the nodes of the ad hoc network decreases.

12. Includes a step during which a node of the network that has received a plurality of said advertisement messages refrains from rebroadcasting at least one of those advertisement messages if it is received from a gateway node offering a relatively low QoS.

13. Each node can choose the best path for routing traffic sent by an active source to the gateway taking account of the quality of the link between the gateway node and gateway; and of the quality of the path between it and the gateway node. Thus it manages end-to-end QoS.

\section{ANALYSIS OF DIFFERENT PROPOSALS FOR WANET-INTERNET INTEGRATION}

In IIW, mobile nodes in the Ad Hoc network need global addresses to communicate with the Internet. Also, when mobile nodes move to another area, their subnet changes, and a new IP address has to be obtained, so node mobility and handoffs should be handled properly $[5,6]$. Several solutions have been proposed for WANET-Internet integration (IIW). Most of the proposed solutions require using number of gateways. These solutions vary in the design and functionality of the gateways, number of occurrences, and the routing protocols used within the WANET. Having two interfaces, Internet gateways act as the part of both the Internet and the Ad Hoc network, so they very well understand the Internet protocol (IP) as well as the WANET routing protocol used.

\subsection{Analysis of Different proposals for Internet-WANET Integration using Reactive discovery Approach}

Bin et al. [7] proposed an adaptive Gateway discovery scheme in which the TTL values of Agent Advertisements (GWADV messages) are dynamically adjusted according to the mobile nodes Internet traffic and their related position from Internet Gateways with which they are registered. It achieves good connectivity while keeping overhead costs low when connecting various scale ad hoc network to the Internet.

Nilsson et al. [8] proposed a modified routing protocol AODV in which I-RREQ and I-RREP messages are used to discover the gateway. This solution relies on the signaling of AODV to find an access providing Internet Gateway that is able to distribute a globally routable prefix for the ad hoc network.

Ammari et al. [9] proposed a mobile gateway (MG) based on three-layer approach using both the mobile IP protocol and DSDV Ad Hoc routing protocol (Figure 2). Mobile IP FAs are contained in the first layer; the second layer has MGs and mobile Internet nodes, which are single-hop away from Mobile IP FAs, thus provides Internet connectivity to WANET nodes; the third layer includes all WANET nodes and also visiting mobile Internet nodes that are at least onehop away from MGs. This framework considers using some border WANET nodes (referred to as MGs) to connect the rest of the WANET nodes to the Internet. Based on the distance and the load criteria, MG selects a closest and/or a least loaded FA. WANET nodes select a closest and/or least loaded MG.
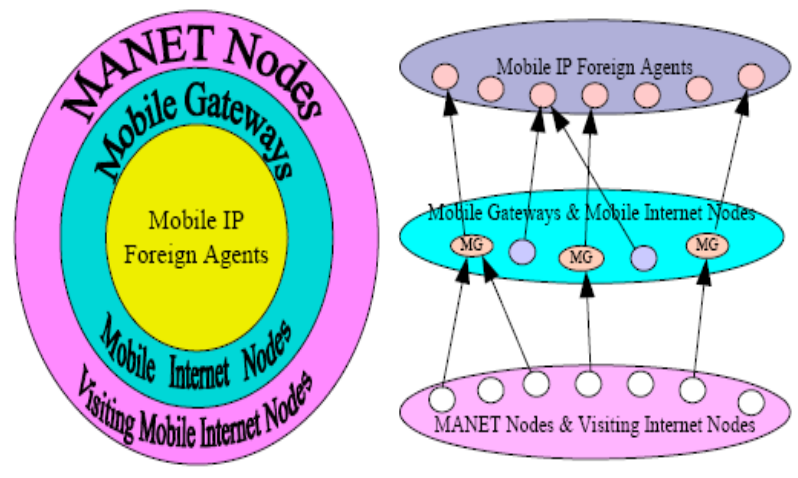

Figure 2. Three layered mobile gateway based architecture

\subsection{Analysis of Different proposals for Internet-WANET Integration using Proactive discovery Approach}

Lei et al [10] integrated the routed (modified RIP) Ad Hoc routing protocol with Mobile IP routing Protocol which resulted in a combined routing table and also enabled FAs to participate in the Ad Hoc network routing.

Xie et al. [11] provided global bi-directional WANET-Internet connectivity by using an enhanced DSDV (EDSDV) protocol for WANETs. The EDSDV protocol integrates with Mobile IP $[12,10]$ by using FA as the mobile IP proxy. Mobile IP protocol provides the global mobility for a mobile host to access Internet resources while visiting a foreign network. Figure 3 shows the proposed protocol, that includes three components: enhanced DSDV routing protocol, mobile IP proxy and connection management.

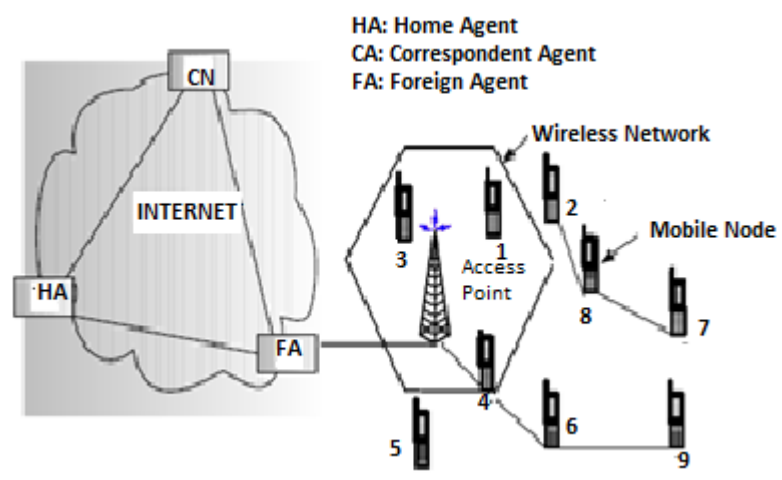

Figure 3. Xie et al. [11] WANET- Internet integration architecture

In [13], E.M. Belding-Royer et al. proposed a scheme which has a proactive agent solicitation procedure with AODV packet sent to the Internet. In addition, it is capable of packet routing using default routing of FA. However, this proposal does not consider the selection between multiple FAs. Also, it delays the connection setup time because this proposal first need to conclude that the destination is not within the WANET prior to a mobile node can use the FA.

Rosenschon et al. [14] proposed a method in which gateway periodically sends HELLO messages that also contain PROAGW option. This option has all information about the gateway that is needed to set up a route to it. The mobile node receives multiple answers if multiple gateways are available; therefore it has to make a decision which gateway it should use. Parameters like hop count, congestion, overload, 
available bandwidth, delay etc. can be considered for the selection of best Internet gateway. But this method floods the WANET with routing messages.

C. Jelger et al. [15] proposed a mechanism where gateways send periodical messages for the auto-configuration of global addresses so as to inform about its existence as well as the prefix it processes. As multiple gateways may be available in the ad hoc network, the nodes may receive multiple autoconfiguration messages originated at different gateways. Forwarding to all of them can cause an excessive load, therefore one gateway will be chosen by a mobile node based on some criteria, and updated message will be sent. By this method, mobile nodes can create a continuous path to the gateway and the same prefix is shared by all of the nodes composing that particular path.

Khan et al. [16] proposed an effective proactive gateway discovery algorithm using modified AODV protocol that takes into account the routing queue length in addition to the minimum hop count metric for selecting and updating the path to the gateway on the request of the mobile node. It facilitates efficient handoff from one gateway to another, and thus maintaining continuous connectivity to the fixed host. Also, these two metrics are not only used to discover the routes to the gateway but also for the routing in the local ad hoc domain among the ad hoc host. This reduces the delay along the path. Further, the occupancy level of each of the node is updated every short interval of time. This updated occupancy is sent to all the neighbors within the radio transmission range within the hello packet. This concept increases the throughput by choosing the less congested paths and reduces the routing overhead.

$L(s, d, h)=$

$\{\min \{\boldsymbol{L}(p, h): s \stackrel{p}{\rightarrow} d, h: \boldsymbol{H}(s, d)\}$ if there is a path from $s$ to $d$

$$
\infty \quad \text { otherwise }
$$

where $\mathrm{L}$ is the packet load along the path, $\mathrm{s}$ is the source(S) node, $\mathrm{d}$ is the destination (D) node, $\mathrm{h}$ is the number of hops between $S$ and $D$, and $p$ is the path between the $S$ and $D$. This will select a path having the minimum packet load and the minimum number of hops.

Khan et al. [17] proposed a method integrating Efficient DSDV (Eff-DSDV) protocol and mobile IP coordinating with each other to provide Internet connectivity. In this, one of the WANET nodes is considered as a Mobile IG (MIG) which runs the Eff-DSDV protocol and also takes care of the addressing mechanisms to ensure the transfer of packets between WANET and Internet. The advantage is that it does not require the flooding of the gateway advertisements for registration of mobile nodes with MIG. It also overcomes the drawback of conventional DSDV by reducing the packet loss due to broken links.

\subsection{Analysis of Different proposals for Internet-WANET Integration using hybrid discovery Approach}

Ratanchandani et al. [18] proposed a hybrid gateway discovery approach to discover gateways that limit the effects of broadcast overhead where AODV and two Mobile IP [12, 10] foreign agents are used to interconnect WANET and the Internet. Mobile nodes use an arbitrary address within the WANET and use a COA for external communication, as specified by Mobile IP. MIPWANET Cell Switching (MMCS) algorithm [5] is used to switch between foreign agents (FA). Its performance depends on the Time-To-Live
(TTL) value, which is set for a particular scenario and network conditions. However, the TTL of the foreign agent's advertisements is limited to only a few hops. Thus, only mobile nodes that are close to one of the foreign agents receive the agent advertisements, but distant nodes have to solicit advertisements reactively. Intermediate nodes are permitted to reply with agent advertisements on a solicitation and also to eavesdrop and cache agent advertisement information that is sent by unicast to the requesting mobile node. Limitations: 1) The set TTL value is not suitable to a generic scenario and only suits a certain scenario with certain number of nodes and topology size. 2) The way of sending Agent solicitation may lead to a long delay for reactive gateway discovery. In brief, the reactive gateway discovery is not efficient, and suffers from the long delay.

Sun et al. [6] discussed the performance of the integration of AODV and Mobile IP. Mobile nodes are enabled to obtain Internet connectivity when one or more nodes are within direct transmission range of FA or more specifically an Internet gateway IG/Access Router. In their approach, an Ad Hoc network is connected to FA, which basically has the same functionality as an IG. IG assigns a global prefix for the Ad Hoc network. While AODV is used for route discovery and maintenance within WANET, Mobile IP provides mobile nodes with care-of-addresses. However, handoff occurs only if a mobile node has not heard from its FA for more than one beacon interval, which is the time between two successive agent advertisements, or its route to a FA has become invalid.

Broch et al. [19] proposed a solution for the integration of dynamic source routing (DSR) protocol and Mobile IP. They introduced a border router, which has two interfaces. Mobile nodes in an Ad Hoc network are assigned home addresses from a single network. The nodes within range of the FA act as gateways between the Ad Hoc network and the Internet. FAs are responsible for connecting WANET with the Internet. As it uses reactive approach, its discovery is done only when required.

Jonsson et al. [5] identified the benefits of choosing the closest access point to the Internet generating less traffic in the network, which results in lower delays and fewer dropped packets and proposed hop count based gateway selection algorithm. They assumed that a mobile node that wants Internet access has been assigned a valid home address on Internet [6]. They proposed MIPMANET method based on AODV, which provides Internet access by using reverse tunneling and Mobile IP with FA care-of addresses. Figure 4 depicts its layered architecture. Mobile nodes that want Internet access register with FA and tunnel all packets destined for the Internet to the registered FA, which in turn forward the packets to the destination in the Internet. The hosts that do not require Internet access see the Ad Hoc network as a standalone network. The tunneling approach also enables MIPMANET to incorporate the default route concept into on-demand routing. AODV is used within the MANET and delivers packets between mobile nodes and FA. If the node is at least two hops closer to the new one, MIPMANET allows handoff, i.e. a visiting node can switch from its current FA to a new one. MMCS algorithm is used to determine when mobile nodes in MANET should register with a new FA.

Ruiz et al. [20] proposed an "adaptive gateway advertisement" with a dynamically adjustable TTL but periodicity of the GW_ADV message is not adjusted dynamically. Maximal benefit coverage algorithm is used by gateways to set the TTL value of their GW_ADV messages. Maximal benefit used to determine the most appropriate TTL 
value for the GW_ADV message is the ratio of cost of flooding the whole ad hoc network with GW_ADV messages and the cost of providing internet connectivity without sending a single GW_ADV message. They derived routing overheads for the proactive, reactive and hybrid methods. However, no deep investigation is done as performance is not analyzed with varying interval times and no test of scalability is done and did not give the results for gateway discovery and handover times.

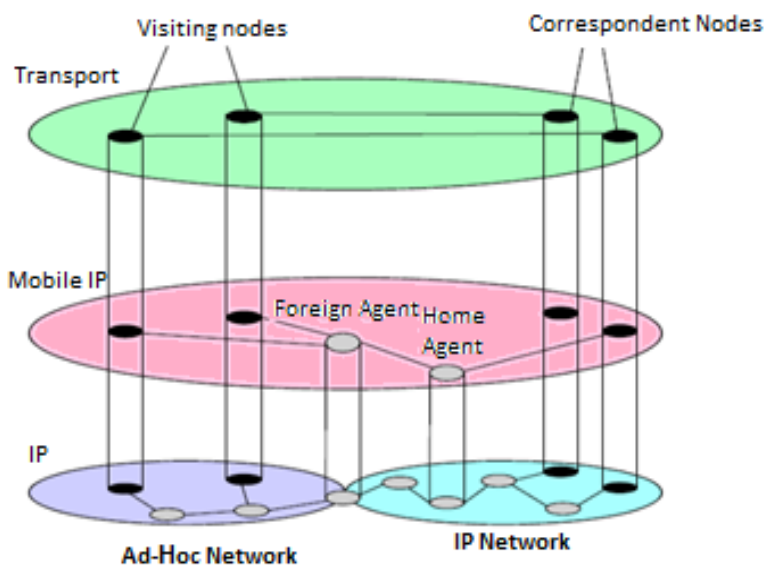

Figure 4. MIPMANET Conceptual view

Tseng et al. [21] proposed a dynamic mobile agent service coverage scheme for integrating DSDV and Mobile IP. Here WANETs are treated as Internet subnets (Figure 5). The existence of each home agent (HA)/ (FA) is known only up to $\mathrm{N}$ wireless hops away from the agents and the mobile nodes outside this range are required to broadcast agent solicitation messages to search for the gateway. The mobile node uses only the number of physical hops metric to determine the ideal HA/FA to connect with. This paper does not evaluate the system or provides a comparison to other solutions, so no thorough investigation.

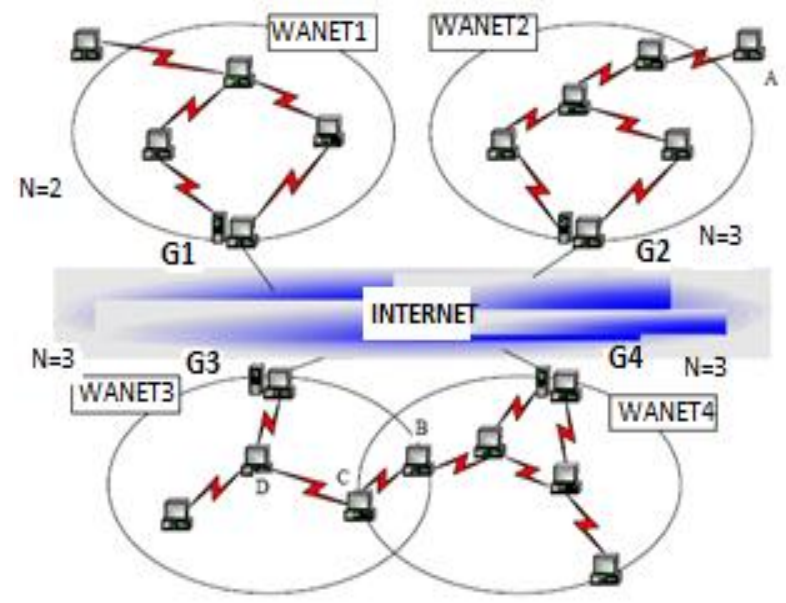

Figure 5. Tseng et al. [21] network architecture extending each access point to a WANET

Kumar et al. [22] proposed the combination of periodic advertisement and adaptive advertisement schemes for AODV. Node can either send solicitations or may depend on the periodic gateway advertisement to obtain information about the available gateways. To make efficient use of this periodic Advertisement, it limits advertisement flooding area, and interval of sending periodic advertisement depends on mobility and topological changes. It uses TTL of the GWADV messages as a parameter to adjust depending on the network conditions. The gateways will keep track of the number of hops for the location of its active sources. The next TTL value to be used for GWADV message is determined using "Maximal Benefit Coverage" heuristic algorithm [20].

Table 1: Summary of Features of Existing Proposals

\begin{tabular}{|c|c|c|c|c|}
\hline $\begin{array}{c}\text { Gateway } \\
\text { Discovery } \\
\text { Approach }\end{array}$ & $\begin{array}{c}\text { Proposed } \\
\text { Architecture } \\
\text { / scheme }\end{array}$ & $\begin{array}{l}\text { Ad Hoc } \\
\text { Routing } \\
\text { Protocol }\end{array}$ & $\begin{array}{c}\text { Multiple } \\
\text { Gateways } \\
\text { Support }\end{array}$ & $\begin{array}{c}\text { Mobile } \\
\text { IP } \\
\text { Support }\end{array}$ \\
\hline \multirow{3}{*}{$\begin{array}{l}\text { REACTIVE } \\
\text { DISCOVERY } \\
\text { APPROACH }\end{array}$} & Bin et al. [7] & AODV & YES & YES \\
\hline & $\begin{array}{c}\text { Nilsson et al. } \\
{[8]}\end{array}$ & AODV & - & YES \\
\hline & $\begin{array}{c}\text { Ammari et } \\
\text { al. [9] }\end{array}$ & $\overline{\text { DSDV }}$ & YES & YES \\
\hline \multirow{7}{*}{$\begin{array}{l}\text { PROACTIVE } \\
\text { DISCOVERY } \\
\text { APPROACH }\end{array}$} & Lei et al [10] & $\begin{array}{l}\text { Modified } \\
\text { version } \\
\text { of RIP }\end{array}$ & YES & YES \\
\hline & $\begin{array}{c}\text { Xie et al. } \\
\text { [11] }\end{array}$ & DSDV & NO & YES \\
\hline & $\begin{array}{l}\text { E.M. } \\
\text { Belding- } \\
\text { Royer et } \\
\text { al.[13] }\end{array}$ & AODV & NO & YES \\
\hline & $\begin{array}{l}\text { Rosenschon } \\
\text { et al. [14] }\end{array}$ & AODV & YES & NO \\
\hline & $\begin{array}{l}\text { Jelger et } \\
\text { al.[15] }\end{array}$ & - & YES & NO \\
\hline & $\begin{array}{c}\text { Khan et al. } \\
\text { [16] }\end{array}$ & AODV & YES & NO \\
\hline & $\begin{array}{c}\text { Khan et al. } \\
\text { [17] }\end{array}$ & DSDV & YES & YES \\
\hline \multirow{9}{*}{$\begin{array}{c}\text { HYBRID } \\
\text { DISCOVERY } \\
\text { APPROACH }\end{array}$} & $\begin{array}{l}\text { Ratanchanda } \\
\text { ni et al. [18] }\end{array}$ & AODV & YES & YES \\
\hline & Sun et al. [6] & AODV & YES & YES \\
\hline & $\begin{array}{c}\text { Broch et al. } \\
\text { [19] }\end{array}$ & DSR & NO & YES \\
\hline & $\begin{array}{c}\text { Jonsson et } \\
\text { al.[5] }\end{array}$ & AODV & YES & YES \\
\hline & $\begin{array}{l}\text { Ruiz [20] } \\
\end{array}$ & AODV & $\mathrm{NO}$ & $\mathrm{NO}$ \\
\hline & $\begin{array}{c}\text { Tseng et al. } \\
\text { [21] }\end{array}$ & $\overline{\mathrm{DSDV}}$ & YES & YES \\
\hline & $\begin{array}{c}\text { Kumar et } \\
\text { al.[22] }\end{array}$ & AODV & YES & NO \\
\hline & $\begin{array}{c}\text { Bin et } \\
\text { al.[23] }\end{array}$ & AODV & YES & NO \\
\hline & $\begin{array}{l}\text { Misbahuddin } \\
\text { et al.[24] }\end{array}$ & - & YES & NO \\
\hline \multirow{3}{*}{$\begin{array}{c}\text { ALL THREE } \\
\text { DISCOVERY } \\
\text { APPROACHES }\end{array}$} & $\begin{array}{l}\text { Hamidian et } \\
\text { al. }[25]\end{array}$ & AODV & YES & NO \\
\hline & $\begin{array}{l}\text { Moshrify et } \\
\text { al. [26] }\end{array}$ & AODV & YES & NO \\
\hline & $\begin{array}{c}\text { Kumar et } \\
\text { al.[27] }\end{array}$ & AODV & YES & NO \\
\hline
\end{tabular}

The number of nodes communicating with the Internet and the number of intermediate nodes (AF nodes) are to be determined. Expression (4.1) gives Total AF nodes for all the sources communicating with the Internet through the gateway.

Total_AF_nodes $=\sum_{1}^{\text {nextTTL }>1} N s(T T L-1)$ 
where Ns are the number of sources communicating with the Internet that are TTL hops away from the gateway. Expression (2) is used when the nextTTL value $>1$. When nextTTL value $=1$, it is preferable to perform an adaptive advertisement as sources are a single hop away from IG. Exp. (4.2) counts Regulated Mobility Degree (RMD) to compute the benefit of performing an adaptive advertisement. Here NS is the total number of sources to be covered by GWADV message.

$R M D=\frac{N S}{\text { Total_AF_nodes }}$

The unidirectional links and the blindly rebroadcasting of gateway discovery packets during ad hoc nodes discovery degrades the Internet connectivity. So to enhance it, Bin et al. [23] proposed an internetworking algorithm with adaptive mechanisms. It exchanges Hello packets identical to that of AODV also containing symmetric and asymmetric neighbor list fields. It discards unidirectional links from route computations and redundant and unnecessary rebroadcasting of the broadcast packets are repressed simultaneously in the procedure of gateway discovery. Also, the dynamic adjustment of the scope of the gateway advertisement packets and the intervals of sending Hello packets according to the change in network conditions reduces a lot of unnecessary overhead.

Misbahuddin et al. [24] developed a modified hybrid gateway discovery mechanism by assigning dynamic value to TTL by using dynamic clusters based mobile gateways. It increases the Internet access reliability and raises the network performance. It gives high data delivery ratio and low average delay. In the proposed architecture (Fig. 6), mobiles are noted as either mobile nodes (MN) or mobile gateway. MNs are bundled into different clusters based on several parameters like their location, moving directions etc. Clustering enhances effective broadcasting and the relaying of messages, increases stability of MNs and reduces signaling overhead.

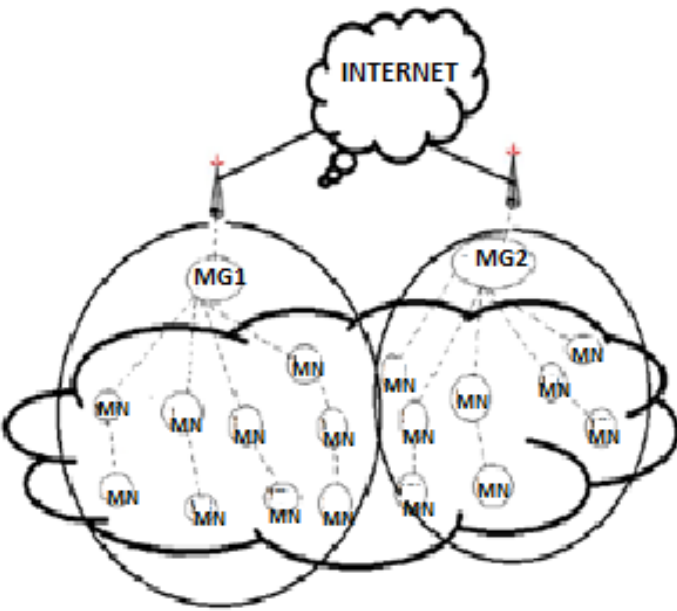

Figure 6. Misbahuddin et al.[24] proposed architecture for Dynamic cluster based adaptive gateway Discovery mechanism in IIW

\subsection{Analysis of Different proposals for Internet-WANET Integration using All Three discovery Approaches}

Hamidian et al. [25] proposed a solution to provide Internet connectivity to ad hoc networks by modifying the AODV routing protocol. AODV RREQ and RREP are extended with "I" flag to locate the fixed node. If $\mathrm{MN}$ does not receive any route replies after one network-wide search, it assumes the destination to be a fixed node located in the Internet and delivers the packets through a gateway. Three methods for gateway discovery for a $\mathrm{MN}$ to access the Internet are investigated: proactive, reactive and hybrid using the number of hops to the gateway as the metric for the gateway selection.

Hossam El-Moshrify et al. [26] proposed a solution in which MNs can access the Internet via a stationary gateway node or access point. Three proposed approaches for Internet gateway discovery are implemented and investigated. It studies and compares the effects of the mobile terminals speed and the number of gateways on the network performance. $\mathrm{MN}$ uses no load balancing approach to efficiently discover IG.

Kumar et al. [27] proposed a modified AODV routing protocol to offer enhanced Internet connectivity. All three Internet discoveries are thoroughly investigated to provide inter- connectivity. Average end-to-end delay, packet loss, throughput and average jitter are compared for performance evaluation. Authors simulations showed that when no. of sessions and data rate is less, all the three Internet gateway discoveries provides higher and more stable throughput, lower packet loss and end-to-end delay. But as the number of sessions and data rate increases, more links break occur due to congestion and buffer overflow, thus lowers throughput. As traffic increases, Average end-to-end delay and packet loss increases, but CBR decreases in all the three discoveries.

Table 1 outlines some of the main features of proposed architectures.

\section{CONCLUSIONS AND FUTURE SCOPE}

This paper throws light on the various issues and solutions proposed by various researchers for gateway discovery for internet connectivity in WANETs. MNs can connect to fixed or mobile Internet gateways. Several approaches have been proposed for WANET-Internet integration in recent years. As multiple gateways is not considered in some of the existing proposals and consequently lack mechanisms for load balancing and scalability which may cause a bottleneck of the gateway, so we have reported its many technical solutions proposed by various researchers. Also, we have observed and analyzed all three approaches for gateway discovery. The reactive approach has less overhead, but produces long delay. The proactive gateway discovery has high overhead, lacks load balancing and wastes enormous network resources due to the frequent advertisement flooding but less delay. Hybrid gateway discovery has a significant disadvantage that some roaming nodes beyond the TTL scope of the Internet gateway may never receive gateway advertisements and also cannot update registrations with their home agents despite having registered with the gateway. Hybrid approach is useful only when a limited number of nodes in the ad hoc network want to access the Internet; else a proactive approach is favored. We also studied some adaptive gateway discovery mechanisms in heterogeneous networks that ideally must address both the issues of dynamically adjusting the TTL value as well as the periodicity of GWADV messages.

Roadblocks to its implementation have been largely overcome in recent years due to the work of several different groups. But there are some areas like an optimal Internet gateway selection, network authentication, and security mechanisms and providing high Quality of Service (QoS), load balancing, scalability, avoiding dead zones and seamless roaming etc. that can be further researched. Adaptive and mobile Internet gateway discovery mechanism can be an interesting future research topic. This paper can serve as a base for extending the gateway discovery approaches with consideration to QoS. 
Also, more work can be done in the direction using dynamic TTL value for hybrid gateway discovery for integrating Internet with WANET.

\section{REFERENCES}

[1] RWakikawa, J.Malinen, C.Perkins, A.Nilsson, and A.Tuominen, "Internet Connectivity for Mobile Ad Hoc Networks,'Internet-Draft, draft-wakikawa-WANETglobal6-02.txt, Nov. 2002

[2] S.baragani, M.Conti, I, Stujmenovic,"Mobile Ad Hoc Networking”, John Willey \& Sons ,IEEE Press, 2004

[3] S.Sarkar,T.G.Basavaraju,C. Puttamadappa,"AD Hoc Mobile Wireless Network:Principles, Protocols \& Applications", Auerbach Publication, 2007

[4] U.Javaid, Djamal-Eddine, " Methods and devices for discovering a gateway and for routing towards said gateway in a hybrid wireless network, "Patent No.:US 8,605,621 B2, Date of Patent: Dec. 10, 2013

[5] U. Jonsson, F. Alriksson, T. Larsson, P. Johansson, and G.M. Maquire, "MIPWANET: Mobile IP for Mobile Ad Hoc Networks,"(MobiHoc 2000), pp. 75-80, August 1999.

[6] Y. Sun, E.M. Belding Royer, C.E. Perkins," Internet Connectivity for Ad Hoc Mobile Networks," IJWIN, Special Issue on WANETs: Standards,Research,Applications (2002).

[7] S. Bin, S. Bingxin, L. Bo, H. Zhonggong, and Z. Li,"Adaptive Gateway Discovery Scheme for Connecting Mobile Ad Hoc Networks to the Internet,"Proceedings of International Conference on Wireless Comm., Networking and Mobile Computing,vol.2,pp.795-799, 2005.

[8] A.Nilsson,C.Perkins,A.Tuominen, R.Wakikawa, J. Malinen, AODV and IPv6 internet access for ad hoc networks, SIGMOBILE Mobile Computer Comm. Review6(3)(2002).

[9] H. Ammari, H. El-Rewini, Integration of mobile ad hoc networks and the internet using mobile gateways, Proceedings of the $18^{\text {th }}$ IPDPS, 2004

[10] Lei H., Perkins C., "Ad Hoc Networking with Mobile IP," in Proceedings of 2nd European Personal Mobile Comm. Conference. (1997).

[11] B. Xie, A. Kumar,"Integrated connectivity framework for Internet and Ad hoc Networks," First International Conference on Mobile Ad hoc and Sensor Systems, October 2004

[12] J. D. Solomon, "Mobile IP: The Internet Unplugged," PH PTR, 1998.

[13] E.M. Belding-Royer, Y. Sun, C.E. Perkins, "Global Connectivity for IPv4 Mobile Ad Hoc Network", IETF Internet-Draft,draft-ietf-WANET-globalv4-00.txt, November 2001

[14] M.Rosenschon, T.Manz, J.Habermann, V.Rakocevic ,"Gateway Discovery Algorithm for Ad-Hoc networks using HELLO Messages",IWWAN 2005, London, May 2005.
[15] C. Jelger , T. Noel, A. Frey, "Gateway and Address Auto-configuration for IPv6 Ad Hoc Networks," IETF internet-Draft, draft-jelger-WANET-gateway-autoconfv6- 02.txt,April 2004

[16] K.U.Khan, A. V Reddy, R.U. Zaman, M.Kumar "An Effective Gateway Discovery Mechanism in an Integrated Internet-WANET (IIM)", 2010 International conference on advances in computer engineering.

[17] K..Rahman Khan, A V Reddy and R.Zaman,"An Efficient Integrated Routing Protocol for Interconnecting Mobile Ad Hoc Network and the Internet,'IJCEE, vol. 1, No. 1, pp. 32-39, April 2009.

[18] P. Ratanchandani, and R. Kravets, "A Hybrid Approach to Internet Connectivity for Mobile Ad Hoc Networks," in Proceedings of the IEEE WCNC 2003, New Orleans, USA

[19] J. Broch, D.A. Maltz, and D.B. Johnson, "Supporting Hierarchy and Heterogeneous Interfaces in Multi-Hop Wireless Ad Hoc Networks," in Proceedings of the IEEE International Symposium on Parallel Architectures, Algorithms, and Networks, Perth, Western Australia, pp.370-375, June 1999.

[20] P.Ruiz, A.Gomez-Skarmeta, "Enhanced Internet Connectivity for hybrid Ad Hoc networks through adaptive gateway discovery," International Conference on Local Computer Networks, Tampa, Florida, November 2004.

[21] Y. C.Tseng, C. C. Shen, and W. T. Chen , "Mobile IP and Ad Hoc Networks: An Integration and Implementation Experience,", Department of Computer Science and Information Engineering, Chaio Tung University, Hsinchu, Taiwan, 2003.

[22] V.kumar, M.Misra" An Efficient Mechanism for Connecting WANET and Internet through Complete Adaptive Gateway Discovery ” IEEE 2006

[23] S.Bin, H.Yangcheng, H.Zhonggong,“ Optimization Gateway Discovery Mechanisms for Hybrid Ad Hoc Networks", IEEE, 2011

[24] M.Misbahuddin, M.Haq, Atif, R.U.Zaman, "Dynamic Cluster based adaptive Gateway Discovery mechanisms in an Integrated Internet-WANET", IJARET,Volume 4, Issue 3, April 2013, pp. 226-232

[25] A. Hamidian, U. Korner, and A. Nilsson, "A Study of Internet Connectivity for Mobile Ad Hoc Networks in NS2", Department of Communication Systems, Lund Institute of Technology, Lund University, January 2003.

[26] H.E.Moshrify， M. A. Mangoud, M.Rizk, "Gateway Discovery in Ad Hoc On-Demand Distance Vector(AODV) Routing for Internet Connectivity," 24th NRSC, March 13-15,2007,Faculty of Engineering, Alexandria University Alexandria 21544., Egypt.

[27] R.Kumar, M.Misra, A.K.. Sarje, “ A Simulation Analysis of Gateway Discovery for Internet Access in Mobile Ad Hoc Networks," International Journal of Information Processing (ISSN 0973-8215) Vol. 2, pp. 52-64, 2008. 